undefined, association with deterioration in cognitive function 6 months after CAS has been reported. ${ }^{4}$ Currently, no protection device can completely prevent distal embolization. Furthermore, dislodgement of embolic debris can occur during guidewire manipulation and protection device positioning or removal. Determining the risk factors for embolization could lead to a better patient selection, improving outcomes of CAS.

Unstable, echolucent atherosclerotic plaques have been associated with increased embolic potential and increased risk of stroke in CAS. ${ }^{5}$ This study confirms the increased embolic potential, because we found a significantly higher number of embolic particles when CAS was performed in the first weeks after symptoms and a significantly higher number of particles in atheromatous plaques.

Older age, hypertension, hypercholesterolemia, coronary artery disease, symptomatic lesions, number of balloon dilations, and stent diameter have unequivocally been associated with increased embolic potential. In this study, we found only smoking to be negatively correlated with the number of dislodged particles during CAS. A possible explanation for this finding is that plaque composition in smokers differs from nonsmokers because of different etiology, and these plaques could be more stable.

Because of the method of histologic processing and section staining, not all filters containing embolic debris appeared in the qualitative analysis. In addition to the small sample size, this is a limitation of this study.

\section{CONCLUSIONS}

The detailed analysis of embolic debris and the findings that lesion characteristics and timing of intervention seem to be the most important factors influencing distal embolization during CAS and contribute to further understanding the mechanism of embolization and better patient selection.

\section{References}

1. Flach HZ, Ouhlous M, Hendriks JM, van Sambeek MRHM, Veenland JF Koudstaal PJ, et al. Cerebral ischemia after carotid intervention. J Endovasc Ther. 2004;11:251-7.

2. Bonati LH, Jongen LM, Haller S, and the ICSS-MRI study group. New ischaemic brain lesions on MRI after stenting or endarterectomy for symptomatic carotid stenosis: a substudy of the International Carotid Stenting Study (ICSS). Lancet Neurol. 2010;9:353-62.

3. Timaran CH, Rosero EB, Martinez AE, Ilarraza A, Modrall JG, Clagett GP. Ath erosclerotic plaque composition assessed by virtual histology intravascular ultrasound and cerebral embolization after carotid stenting. J Vasc Surg. 2010;52: 1188-94.

4. Altinbas A, van Zandvoort MJ, van den Berg E, Jongen LM, Algra A, Moll FL, et al. Cognition after carotid endarterectomy or stenting: a randomized comparison. Neurology. 2011;77:1084-90.

5. Topakian R, Strasak AM, Sonnberger M, Haring HP, Nussbaumer K, Trenkler J, et al. Timing of stenting of symptomatic carotid stenosis is predictive of 30-day outcome. Eur J Neurol. 2007;14:672-8.

\title{
Is there any benefit in using awake anesthesia with thoracic epidural in thoracoscopic talc pleurodesis?
}

\author{
Eugenio Pompeo, MD, ${ }^{\mathrm{a}}$ and Mario Dauri, MD, ${ }^{\mathrm{b}}$ for the Awake Thoracic Surgery Research Group, \\ Rome, Italy
}

\section{Supplemental material is available online.}

General anesthesia is commonly used for video-assisted thoracic surgery (VATS) with talc pleurodesis, although use of regional anesthesia in the awake patient might

\footnotetext{
From the Departments of Thoracic Surgery ${ }^{\mathrm{a}}$ and Anesthesiology and Intensive Care, ${ }^{\mathrm{b}}$ Policlinico Tor Vergata University, Rome, Italy.

Disclosures: Authors have nothing to disclose with regard to commercial support.

The Awake Thoracic Surgery Research Group is listed in the Appendix.

Received for publication Dec 5, 2012; revisions received March 19, 2013; accepted for publication March 25, 2013; available ahead of print April 22, 2013.

Address for reprints: Eugenio Pompeo, MD, Section of Medical and Surgical Lung Diseases, Department of Biomedicine and Prevention, Tor Vergata University,

Via Montpellier 1, 00133 Rome, Italy (E-mail: pompeo@med.uniroma2.it).

J Thorac Cardiovasc Surg 2013;146:495-7

$0022-5223 / \$ 36.00$

Copyright (c) 2013 by The American Association for Thoracic Surgery

http://dx.doi.org/10.1016/j.jtcvs.2013.03.038
}

improve outcomes. ${ }^{1,2}$ The purpose of this randomized study is to compare the hospital discharge of patients undergoing VATS with talc pleurodesis using thoracic epidural or general anesthesia.

\section{CLINICAL SUMMARY}

Forty patients with malignant pleural effusion were randomized to undergo VATS with talc pleurodesis by awake anesthesia with thoracic epidural (awake group, $\mathrm{N}=20$ ) or general anesthesia and 1-lung ventilation (control group, $\mathrm{N}=20$ ) (ClinicalTrials.gov registration No. NCT01469728). The study was approved by the Policlinico Tor Vergata University ethical committee, and written informed consent was obtained by the patients. Eligibility criteria are summarized in Table E1.

In the awake group, the thoracic epidural catheter was inserted at the T4 level for continuous infusion of ropivacaine 

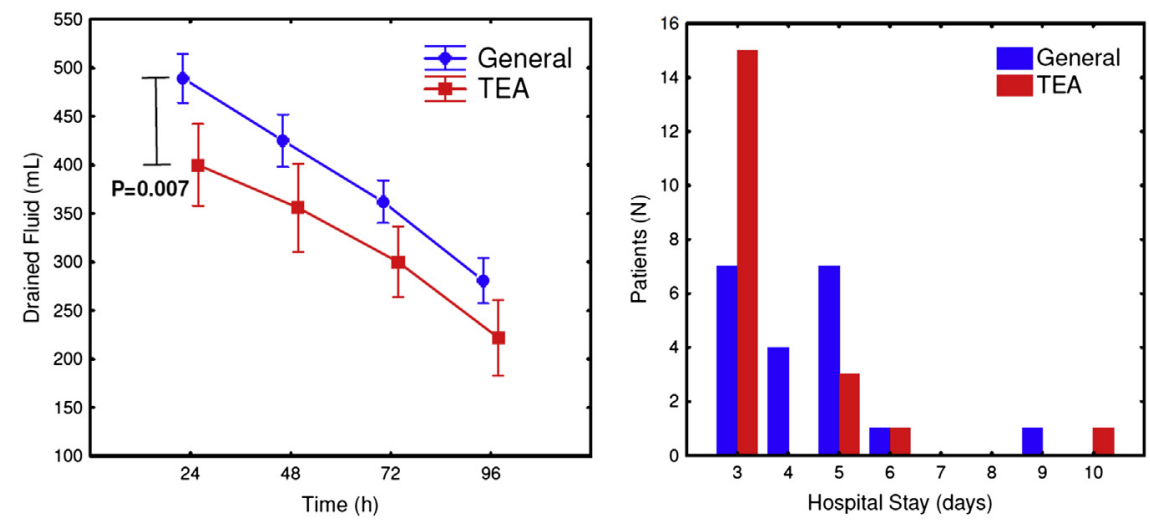

FIGURE 1. Daily amount of drainage volume (left) and histogram detailing hospital stay (right) in the study groups. TEA, Thoracic epidural anestesia.

plus sufentanil. Patients remained conscious during the procedure.

In the control group, general anesthesia was induced by propofol with a single dose of cisatracurium for muscle relaxation. One-lung ventilation was achieved by a left-sided double-lumen tube.

The surgical procedure was performed through a single flexible trocar. After evacuation of the effusion, $5 \mathrm{~g}$ of asbestos-free talc powder was insufflated under visual control onto the entire pleural surface. After surgery, the chest tube was placed on $20 \mathrm{cmH}_{2} \mathrm{O}$ suction, and in the awake group, the epidural catheter was removed within 24 hours. Criteria for discharge were standardized and included 24/hour fluid drainage less than $200 \mathrm{~mL}$. During the follow-up, a chest $\mathrm{x}$-ray was performed at 1 month.

The primary outcome was early hospital discharge (hospital stay $\leq 3$ days). Secondary outcome measures included assessment of the daily drainage volume, oxygenation measures, postoperative pain, in-hospital charges, need for redo drainage, and survival. Patients' satisfaction with the assigned anesthesia protocol was graded according to an ordinal scale ranging from 4 (optimal) to 1 (unsatisfactory).

TABLE 1. Main results

\begin{tabular}{lccc}
\hline & Awake group & Control group & $\boldsymbol{P}$ value \\
\hline Operative time (min) & $30 \pm 8$ & $29 \pm 5$ & .63 \\
Anesthesia satisfaction (score) & $2.8 \pm 1.0$ & $2.4 \pm 0.7$ & .19 \\
24-h pain VAS (score) & $1.7 \pm 0.5$ & $2.1 \pm 0.8$ & .16 \\
24-h $\mathrm{PaO}_{2} / \mathrm{FIO}_{2}(\mathrm{~mm} \mathrm{Hg})$ & $325 \pm 33$ & $326 \pm 24$ & .96 \\
24-h $\mathrm{PaCO}_{2}(\mathrm{~mm} \mathrm{Hg})$ & $42 \pm 2$ & $42 \pm 3$ & .60 \\
Hospital stay (d) & $3.8 \pm 1.7$ & $4.3 \pm 1.5$ & .07 \\
Early discharge ( $\leq 3 \mathrm{~d})$ & 15 & 7 & .02 \\
Procedure-related cost (euros) & $3015 \pm 1450$ & $3780 \pm 1170$ & $<.00001$ \\
Redo drainage (N) & 2 & 3 & .66 \\
Survival (mo) & 5.1 & 6 & .8 \\
\hline
\end{tabular}

$\mathrm{VAS}$, Visual analogue scale; $\mathrm{PaO}_{2} / \mathrm{FIO}_{2}$, ratio of arterial oxygen tension to fraction of inspired oxygen; $\mathrm{PaCO}_{2}$, arterial carbon dioxide.
For the study purpose, 20 patients per group were calculated to be necessary by a 2-tailed test assuming a 2.5 -fold increase of early discharges using regional anesthesia $(\alpha=0.05$; power $=0.80)$.

The nonparametric Mann-Whitney test and 2-tailed Fisher exact test were used to match averaged data and proportions, respectively. Survival was assessed by the Kaplan-Meier method.

\section{RESULTS}

There were no intergroup differences in demographics and baseline characteristics (Table E2). No patient in the awake group required sedation or conversion to general anesthesia. Hospital mortality included 1 patient in the control group who died of cancer progression.

The proportion of early discharges was significantly greater in the awake group. In addition, the drainage volume 24 hours after surgery and procedure-related costs were better in the awake group, whereas oxygenation, postoperative pain, mortality, effusion recurrence, and survival were comparable between groups (Figure 1 and Table 1).

\section{DISCUSSION}

The most striking result of this study is that VATS with talc pleurodesis performed using awake anesthesia with thoracic epidural resulted in a significantly greater proportion of early discharges than procedures performed using general anesthesia. Reasons underlying such a beneficial effect are conjectural and include better control of the pleural space with more rapid lung reexpansion, which may be due to superior perioperative pain relief or reduced barotrauma and volutrauma under spontaneous ventilation. The more frequent early discharges achieved in the awake group eventually resulted in lower costs, whereas the need for redo drainage and survival were comparable between study groups.

Excellent results have been reported for VATS with talc pleurodesis performed using intercostal blocks and 
sedation. ${ }^{2}$ We prefer epidural anesthesia because it provides a wider thoracic analgesia than local anesthesia and obviates the need for adjunctive sedation. Furthermore, maintenance of consciousness is useful at the end of the procedure when lung reexpansion can be achieved physiologically by the patient through repeated coughing. ${ }^{3}$ Other supposed benefits of epidural anesthesia include increased myocardial blood flow, reduced rate of thromboembolic events, and attenuation of humoral stress response. ${ }^{4}$ On the other hand, epidural anesthesia-related complications, such as the risk of epidural hematoma, must be acknowledged, particularly in patients with coagulation disorders.

Alternative options for management of malignant pleural effusion include bedside talc slurry and use of indwelling catheters placed in the outpatient setting, which hold promise $^{5}$ and should be compared with awake VATS pleurodesis in the near future.

Limitations of our study include the use of a dichotomized continuous variable as a primary outcome measure, which was chosen intentionally to ensure that the trial had sufficient power with a limited, single-center cohort.

\section{CONCLUSIONS}

Awake VATS with talc pleurodesis resulted in a greater proportion of early discharges and eventually lower costs than procedures performed with general anesthesia. Further data from larger studies will be necessary to corroborate our findings.

\section{References}

1. Yim APC, Chan ATC, Lee TW, et al. Thoracoscopic talc insufflations versus talc slurry for symptomatic malignant pleural effusion. Ann Thorac Surg. 1996;62: 1655-8.

2. Katlic MR, Facktor MA. Video-assisted thoracic surgery utilizing local anesthesia and sedation: 384 consecutive cases. Ann Thorac Surg. 2010;90:240-5.

3. Pompeo E, Tacconi F, Mineo D, Mineo TC. The role of awake video-assisted thoracoscopic surgery in spontaneous pneumothorax. I Thorac Cardiovasc Surg. 2007; 133:786-90.

4. Chaney MA. Intrathecal and epidural anesthesia and analgesia for cardiac surgery. Anesth Analg. 2006;102:45-64.

5. Dresler CM, Olak J II, Richards WG, et al. Phase III intergroup study of talc poudrage vs talc slurry sclerosis for malignant pleural effusion. Chest. 2005; 127:909-15.

\section{APPENDIX}

Awake Thoracic Surgery Research Group: Tor Vergata University, Rome, Italy: Eugenio Pompeo, MD (Principal Investigator), Maria Antonietta Ceccon, MD, Ludovica Celidonio, MD, Benedetto Cristino, MD, Mario Dauri, MD, Filippo De Padova, MD, Eleonora Fabbi, MD, Francesca Leonardis, MD, Mario Marino, MD, Tommaso Claudio Mineo, MD (Chief Thoracic Surgery Department), Augusto Orlandi, MD, Paola Rogliani, MD, Alessandro Fabrizio Sabato, MD, Elisabetta Sabato, MD, Cesare Saltini, MD, Federico Tacconi, MD. University La Sapienza, Rome, Italy: Italo Nofroni, BS. 
TABLE E1. Inclusion criteria

Free-flowing unilateral pleural effusion on chest computed tomography occupying at least one third of the hemithorax in patients with recent history of malignancy.

Karnofsky performance status $\geq 50$

ASA score II-III

Acceptance of randomized assignment to the anesthesia protocol

Absence of coagulation disorders (INR $<1.5$ )

No contraindications for thoracic epidural anesthesia

No neurologic or psychiatric disturbance contraindicating awake anesthesia during surgery

$A S A$, American Society of Anesthesiology; INR, international normalized ratio.

TABLE E2. Demographics and baseline data

\begin{tabular}{lccc}
\hline & Awake group & Control group & $\boldsymbol{P}$ value \\
\hline Age, y & $67 \pm 12$ & $67 \pm 10$ & .95 \\
$\mathrm{M} / \mathrm{F}$ ratio & $13 / 7$ & $10 / 10$ & .34 \\
Lung cancer/other histology & $6 / 14$ & $11 / 9$ & .20 \\
$\mathrm{PaCO}_{2}(\mathrm{~mm} \mathrm{Hg})$ & $42 \pm 3$ & $41 \pm 3$ & .44 \\
$\mathrm{PaO}_{2} / \mathrm{FIO}_{2}$ (mm Hg) & $328 \pm 33$ & $328 \pm 26$ & .97 \\
$\mathrm{FEV}_{1}(\mathrm{~L})$ & $1.94 \pm 0.7$ & $1.92 \pm 0.5$ & .91 \\
$\mathrm{FEV}_{1}$ (\% predicted) & $79 \pm 13$ & $78 \pm 14$ & .85 \\
$\mathrm{FVC}(\mathrm{L})$ & $2.6 \pm 0.7$ & $2.7 \pm 0.7$ & .72 \\
$\mathrm{FVC}(\%$ predicted) & $79 \pm 14.5$ & $80 \pm 14$ & .85 \\
$\mathrm{FEV} / \mathrm{FVC}$ ratio & $0.73 \pm 0.1$ & $0.71 \pm 0.1$ & .54 \\
$\mathrm{PEF}(\mathrm{L} /$ min) & $300 \pm 45$ & $298.5 \pm 70$ & .90 \\
PEF (\% predicted) & $87 \pm 10$ & $86 \pm 14$ & .21 \\
ASA III (rate) & $12 / 20$ & $11 / 20$ & 1.0 \\
Karnofsky score & $66 \pm 10$ & $67 \pm 10$ & .7 \\
\hline
\end{tabular}

Ordinal variables are expressed as mean \pm standard deviation. $\mathrm{PaCO}_{2}$, Arterial carbon dioxide; $\mathrm{PaO}_{2} / \mathrm{FIO}_{2}$, ratio of arterial oxygen tension to fraction of inspired oxygen; $F E V_{l}$, forced expiratory volume in 1 second; $F V C$, forced vital capacity; $P E F$, peak expiratory flow; ASA, American Society of Anesthesiology score (range I-IV). 